Anaesthesist 2009 · 58:121

DOI 10.1007/s00101-009-1516-1

Online publiziert: 13. Februar 2009

๑) Springer Medizin Verlag 2009

\author{
R. Gärtner \\ Medizinische Klinik Innenstadt, Universität München
}

\section{Kortikosteroidinsuffizienz bei kritisch Kranken}

\section{Unterschätzte klinische Entität?}

Jeder physische und psychische Stress, insbesondere aber auch jede schwere Erkrankung aktiviert die hypothalamische hypophysäre adrenale Achse. Die Folge ist ein Anstieg des Kortisols im Plasma um ein Vielfaches der Normalwerte. Bei kritisch Kranken ist die Höhe dieses Anstiegs direkt mit der Schwere der Erkrankung korreliert. Ist dieser Kortisolanstieg aber im Verhältnis zur Schwere der Krankheit inadäquat, spricht man von einer relativen Nebenniereninsuffizienz, nach der neuen Nomenklatur von einer Kortikosteroidinsuffizienz bei kritisch Kranken [„critical illness-related corticosteroid insufficiency" (CIRCI); [4]]. Es ist schon lange bekannt, dass diese mit einer signifikant erhöhten Mortalität assoziiert ist.

Die Glukokortikoide gehören zu den klassischen Stresshormonen. Sie haben nicht nur einen wesentlichen Einfluss auf den Glucose-, Protein- und Fettmetabolismus, sondern sie beeinflussen auch das kardiovaskuläre System. Sie steigern die myokardiale Kontraktilität, den Gefäßtonus und erhöhen den Blutdruck, indem sie sowohl die Synthese als auch das Ansprechen von Angiotensin II, Adrenalin und Noradrenalin auf das Gefäßsystem steigern. Sie modulieren die Gefäßpermeabilität und erniedrigen die Produktion von Stickstoffmonoxid (NO) sowie anderen Vasodilatatoren. Bedeutsam ist auch die Wirkung der Glukokortikoide auf das Immunsystem. Sie stimulieren die Produktion von antiinflammatorischen Zytokinen, hemmen die inflammatorische Zytokinfreisetzung sowie die Expression von entzündlichen Mediatoren wie Phospholipase 2, Prostaglandin-Synthetase, Zyklooxygenase 2 und die induzierbare NO-Synthase.
All diese bekannten Effekte der Glukokortikoide belegen eindrücklich die Bedeutung dieser Hormone nicht nur bei körperlichem Stress, sondern gerade auch bei kritisch kranken Patienten. Sie waren die pathophysiologische Grundlage, auf der mehrere Studien durchgeführt wurden, zunächst mit pharmakologischen, sehr hohen Dosen an Glukokortikoiden, insbesondere bei Sepsis und septischem Schock, allerdings mit negativem Erfolg und einer erhöhten Mortalität. Jedoch kann eine nahezu physiologische, stressadaptierte Substitution mit 200-300 mg Hydrokortison zumindest bei einigen Patienten mit septischem Schock den klinischen Verlauf positiv beeinflussen und sogar die Mortalität signifikant senken $[1,5]$.

Das Hauptproblem besteht jedoch in der Diagnose der CIRCI und damit der Identifizierung der Patienten, die von einer Substitutionstherapie profitieren können. Welcher Patient leidet tatsächlich an dieser relativen Insuffizienz der Nebennierenfunktion? Es war zunächst versucht worden, dies laborchemisch zu definieren, wobei man sich nach den Kriterien bei Gesunden richten musste. Diese relative Nebenniereninsuffizienz wurde entweder als ein Kortisolgehalt $<34 \mu \mathrm{g} / \mathrm{l}$ oder ein $\Delta$-Kortisol-Gehalt $<9 \mu \mathrm{g} / \mathrm{l}, 30 \mathrm{~min}$ nach $250-\mu \mathrm{g}-$ Cosyntropin-Injektion definiert $[2,3]$. Neben den methodischen Problemen der Kortisolbestimmung und der zeitlichen Verzögerung, bis die Laborwerte verfügbar sind, stellt sich die Frage, ob diese Grenzwerte tatsächlich für die Praxis sinnvoll sind. Somit ist der neueste Versuch der klinischen Definition eines CIRCI, nämlich das fehlende Ansprechen auf Volumen- und Katecholamingaben, zu begrüßen.
In dem sehr ausführlichen und verständlich dargestellten Beitrag von Briegel et al. in diesem Heft wird umfassend auf die pathophysiologischen Grundlagen und die diagnostischen Probleme der CIRCI eingegangen, die Datenlage der klinischen Studien kritisch diskutiert, und Ausblicke auf die möglichen Indikationen werden gegeben. Der Beitrag stellt die Bedeutung dieser möglicherweise unterschätzten Insuffizienz der Nebennierenrindenfunktion bei kritisch kranken Patienten sehr überzeugend dar.

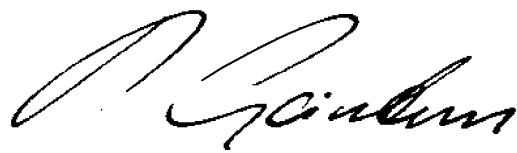

Roland Gärtner

\section{Korrespondenzadresse \\ Prof. Dr. R. Gärtner \\ Medizinische Klinik Innenstadt, Universität München \\ Ziemssenstraße 1, 80336 München \\ roland.gaertner@med.uni-muenchen.de}

\section{Literatur}

1. Annane D, Sébille V, Charpentier C et al (2002) Effect of treatment with low doses of hydrocortisone and fludrocortisone on mortality in patients with septic shock. JAMA 288:862-871

2. Cooper MS, Stewart PM (2007) Adrenal insufficiency in critical illness. J Intensive Care Med 22:348-362

3. Cooper MS, Stewart PM (2003) Corticosteroid insufficiency in acutely ill patients. N Engl J Med 348:727-734

4. Marik PE, Pastores SM, Annane D et al (2008) Recommendations for the diagnosis and management of corticosteroid insufficiency in critically ill adult patients: consensus statements from an International Task Force by the American College of Critical Care Medicine. Crit Care Med 36:1937-1949

5. Sprung CL, Annane D, Keh D et al (2008) Hydrocortisone therapy for patients with septic shock. $\mathrm{N}$ Engl J Med 358:111-124 